\title{
Effects of Sustained, Coordinated Activities Programming in Long-Term Care: The Memory in Rhythm ${ }^{\circledR}$ Program
}

\author{
Iva De Witt-Hoblit' ${ }^{1}$ Mary Neal Miller ${ }^{1}$, Cameron J. Camp ${ }^{2 *}$ \\ ${ }^{1}$ miVision, LLC, West Liberty, Ohio, USA \\ ${ }^{2}$ Center for Applied Research in Dementia Solon, Ohio, USA \\ Email: iva@woh.rr.com,marynealmivision@woh.rr.com, "cameron@cen4ard.com
}

Received 23 December 2015; accepted 25 January 2016; published 28 January 2016

Copyright (C) 2016 by authors and Scientific Research Publishing Inc.

This work is licensed under the Creative Commons Attribution International License (CC BY). http://creativecommons.org/licenses/by/4.0/

(c) $\underset{\mathrm{EY}}{\mathrm{i}}$ Open Access

\begin{abstract}
Beneficial effects of providing engaging activities to long-term care residents have been well documented. However, it is important to determine the effects of activities when providing throughout the day, especially as they related to outcomes salient to administrators. We describe the creation and pilot testing of a sustained, coordinated activities program, Memory in Rhythm ${ }^{\circledR}$ (MIR), which incorporated Montessori-Based Dementia Programming ${ }^{\mathrm{TM}}$, in a skilled nursing facility (SNF). Effects of implementing MIR then were examined in memory care units in 16 aged care centers- 9 SNFs and 7 assisted living residences in Ohio. For these centers, all data were collected over a period of one year before and one year after implementation of MIR. Results indicate that implementation of MIR was associated with reductions in medication use, increased census, decreased employee turnover, decreased wandering and agitation, and increased sleeping at night, eating and capacity for activities of daily living. In the SNFs, increases in RUGS case mix and use of Medicare Part B (rehabilitation services) were noted, while in assisted living implementation also was related to increased amount of time residents who were able to age in place. Implications of these findings are discussed.
\end{abstract}

\section{Keywords}

Dementia, Engagement, Medication Reduction, Agitation, Wandering, ADLs, Employee Turnover, Census, Long-Term Care, Assisted Living

\section{Introduction}

The effects of providing engaging activities to long-term care residents have been well documented. These in${ }^{*}$ Corresponding author.

How to cite this paper: De Witt-Hoblit, I., Miller, M.N. and Camp, C.J. (2016) Effects of Sustained, Coordinated Activities Programming in Long-Term Care: The Memory in Rhythm Program. Advances in Aging Research, 5, 1-8. 
clude increased positive engagement, decreased challenging behaviors, increased positive effect, decreased medication usage, etc. [1] [2]. In practice, however, provision of activities generally is sporadic, and often given a low priority. This is evidenced by routine interruptions of activities for provision of medications, rehabilitation, etc. In addition, a commonly heard complaint on long-term care units is "The residents are fine during an activity, but once it is over the problems come back." The traditional "10 \& 2" activities program, which provides a daily activity at 10 a.m. and 2 p.m., at best is passé and at worst woefully lacking. Such a simple schedule does not meet the engagement needs of most long-term care or senior living residents, much less those with significant memory loss.

This represents a view that activities provided to long-term care residents not only are a low priority, but also should be able to produce effects analogous to that of an antibiotic. Just as an antibiotic generally maintains its effects over a time frame of many hours once it enters the bloodstream, it is assumed that activities ought to maintain beneficial effects long after programming is concluded. This view ignores the role of the physical and social environments on the behavior of residents, and represents a highly mechanistic and inappropriate way of thinking regarding non-pharmacologic treatment modalities, which can be highly effective treatment regimens for persons with dementia and related disorders [1] [3]-[5].

Attempts to counter such attitudes have involved alternative approaches such as person-centered care [5]. These alternative approaches represent a different paradigm, with a different set of assumptions regarding residents and the relationship of residents to the physical and social environments of long-term care. As is often the case, attempts to change paradigms meet with resistance. Administrators of long-term care residences worry about potential unknowns involved with paradigmatic change, and are especially concerned with issues of costs associated with "novel" approaches to care delivery, making the assumption that new approaches will involve additional costs over and above current levels (It is our experience that residences with reasonable staffing patterns typically do not have to add new staffing to have excellent results, as we will discuss shortly.)

One way to help bridge this divide between paradigms, and make it easier for administrators and staff to accept change, is to view activities as a treatment. From this perspective, we may describe activities, like many drugs, as having dose-dependent effects. In addition, we might view activities in long-term care as having a short "half-life." Fortunately, activities are much less prone to unintended negative effects and toxicity than many pharmaceuticals used in long-term care, especially when providing repeatedly over extended periods of time. Activities also can be more effective at reducing or alleviating problematic (i.e., "responsive") behaviors (e.g., repetitive behaviors; wandering; etc.) in residents than pharmaceutical treatments. In addition, we must view activities as living-everything that a resident does legitimately may be considered "activity" (e.g., activities of daily living), and thus the domain and responsibility of all persons who interact with residents is both direct and indirect [6] [7]. Viewed from this perspective, the challenge to administrators becomes one of creating procedures and infrastructure that will enable a treatment with a short half-life that is effective to be delivered frequently enough during the day to provide noticeable and sustained positive results. Now we will describe an approach to delivery of activities created within this context—-the Memory in Rhythm ${ }^{\circledR}$ Program (MIR).

\subsection{Creation of the MIR Program}

MIR was developed by Iva De Witt-Hoblit, LNHA, MRE and Mary Neal Miller, BSN, RN. The basic program was created by De Witt-Hoblit in 1999, to assist residents in an all-Alzheimer's skilled nursing residence of which she was administrator. Even in the infancy of the program, residents experienced reduced agitation and improved engagement in life activities. Later, Miller, who was director of nursing at the nursing residence administered by De Witt-Hoblit, added clinical and restorative nursing portions into the program. Therapeutically, this resulted in enhanced resident independence in activities of daily living, decreased urinary tract infection, and decreased weight loss. From a skilled nursing residence operations perspective, outcomes included enhanced RUGS case mix scores, reimbursements and revenues.

In this particular residence where De Witt-Hoblit and Miller collaborated, there was no designated dementia unit but there were many residents with memory loss issues, primarily dementia. These residents were "lost" in the crowd of other residents. They were agitated, acting out, awake at night, disturbing others, moving furniture and continually wandering. Obviously, there was a need to create interventions for these behaviors, but there also was a large gap in available services. However, Necessity is truly the mother of invention.

Given this great need in the residence, De Witt-Hoblit and Miller decided to implement an intervention pro- 
gram at this residence without a designated unit. Knowing this would take time to implement, they initially started a Sundowner's Program that ran from 3 p.m.-7 p.m. The majority of the challenging behaviors of residents targeted for the program were exhibited in the afternoon, showing that this was the time residents needed the intervention most. Mimicking part of the future MIR, this program began with the high and low energy related interventions:

They were as follows:

- High energy activity such as those requiring expenditure of physical effort

- "Getting-ready-for-supper" activity

- Supper

- After supper, a light cognitive activity.

This Sundowner's program involved eight residents with the greatest needs. The residents chosen had two or more of the following issues:

- Daily agitation

- Daily wandering

- Awake at night

- Significant memory loss/lost as what to do next

- Weight loss.

The criteria used for inclusion in the program were that residents needed to be:

- Ambulatory (walker, cane acceptable)

- Presenting with psychosocial needs that outweighed their physical needs

- Able to participate in programming $75 \%$ of the time.

One STNA (state-tested-nursing assistant) was used to implement the Sundowner's Program. No additional staffing was added. There was not a need to add staffing, as once these residents were brought into a safe, calm and engaging environment, the rest of the staff care givers were freed up to provide care for other residents. Previously, most of the staff members were spending large amounts of their time trying to manage the behaviors of the memory loss residents, minimizing time for being with and caring for other residents.

Within a week, the agitation, wandering and sleeplessness seen in the Sundowner's Program residents were greatly reduced. Other residents stated that they felt more comfortable to come out of their rooms. Visitors and families stated that the residence had an overall calmer ambience after the implementation of the Sundowners program. The program was run 7 days a week, until full implementation of MIR replaced the Sundowner's Program with these residents, and including other residents with responsive/challenging behaviors.

In early 2002, the team implemented MIR into the residence. This was accomplished by setting up an "internal adult day program" within the residence. The team renovated an activity area which initially was two small rooms into a MIR program area. A wall dividing the rooms was removed and the area now included a small dining and living room area to seat 12 persons. In addition, the area included a kitchenette and a bathroom which were dedicated solely to this program.

The program ran from 10 a.m.-6 p.m. The residents came after breakfast and once their activities of daily living were complete. Seven days a week, these residents came to their "special" area, which many came to call "home." Some thought this was their home, some that this was where they worked. They were allowed their own reality.

The following steps were critical to putting the program in place.

- Renovating the activity/program area;

- Educating families and community about the coming program;

- Comprehensive training of frontline staff in the program;

- Selection of a staff member "champion" to oversee program;

- Insisting that the champion would never be assigned to another program or position without the expressed consent of the executive director, and insisting that short staffing would never be a reason for "temporarily" reassigning the program champion.

- Recruiting the most qualified internal caregivers to daily implement the program;

- Training all staff in the concepts of the program along with basic Alzheimer's/Dementia/Memory Loss information;

- Hands-on practice days with staff and residents;

- Rolling out the program in phases (First week—-four residents; Third week—adding four more residents); 
- Using the same criteria for resident selection as for the Sundowner's program.

From the beginning other interventions were utilized, including art, aroma, and pet therapy. The combination of our initial programming and these added interventions resulted in reduced agitation, reduced wandering, reduced medication usage and reduced weight loss.

\subsection{Addition of Montessori-Based Dementia ProgrammingTM}

In fall of 2002, in collaboration with Cameron Camp, PhD., the team added the Montessori-Based Dementia Programming ${ }^{\mathrm{TM}}$ (MBDP) component to MIR. MBDP involves activities based on the Montessori educational method, adapted for use with older adults with dementia. This approach uses techniques based on rehabilitation principles such as breaking down tasks into steps and working on one step at a time, extensive use of external aids to guide performance, emphasizing the use of abilities that are present rather than disabilities, creating materials and procedures to allow success while circumventing deficits, extensive use of materials that involve physical manipulation, and emphasizing the values of respect and dignity for all persons [1] [8] [9].

This addition enhanced the results of the already positive outcomes, as well as producing increased independence with activities of daily living, reduced medication usage, fall reduction and increased length of time a resident could remain in the program. Remarkable results included residents relearning to feed themselves, dress themselves and some regaining speech abilities. (See also [2])

\section{3. "Reflections" Program}

In 2003, the team implemented another MIR program (which was called "Reflections") for residents who had higher physical needs, and who could only participate in the program $50 \%$ of the time. This group experienced an even higher outcome in enhanced ability to feed oneself and improved finger dexterity. The maximum number of residents in Reflections was 14, with one caregiver implementing the program. Activities of Daily Living (ADL) care was provided by other STNA's.

Overtime the MIR and Reflections residents were moved to live in the same section of the residence, although both internal adult day programs stayed in their same location. The team established a separate program area for the Reflections residents to experience their day. The Reflections residents took a much longer rest time in the afternoon.

The basic program of the MIR followed an ebb and flow energy pattern. The day was filled with high physical energy times, high mental energy times, low physical energy times and low mental energy times, alternating based on needs of most residents. Experience showed that most residents have the greatest success when there is at least one high physical energy activity and one high mental energy activity in the morning, a rest and relaxation after lunch, one high physical energy and one high mental energy activity in the afternoon, and a light cognitive stimulation time after supper. Examples of high physical energy activities used are dancing and balloon volleyball. Examples of low physical energy activities used are range of motion exercises and facial massages. Examples of high mental energy activities used are Montessori-based category sorting (e.g., items seen at a circus or not seen at a circus) and a Spelling Bee. Examples of low mental energy activities used are listening to soft music and watching a TV game show.

MBDP [1] [8] [9] fits nicely into MIR, creating successful restorative nursing opportunities and cognitive interventions. Montessori programming can be incorporated in all of the energy level activities, whether high or low. When utilized on a daily basis, Montessori program with MIR results in significant improvements in residents' activities of daily living, as we will demonstrate shortly.

Some very general principles of Memory in Rhythm ${ }^{\circledR}$ Program include the following:

1) First and foremost, consistency and regularity are of upmost importance- the program must be conducted seven days a week without fail.

2) Individuals with cognitive impairments should start the day at essentially the same time and the same way each day. This means choosing a rising time that is realistic 7 days a week. In addition, the initial morning routine should be very consistent (for example, breakfast time, taking their medications, oral care, bathing and or dressing). Best results include providing showers in the morning and evening, prior and after main programming.

3) Consistent hydration is very important and the morning routine should be followed by some sort of noncaffeinated beverage. Hydration should continue throughout the day every two hours during waking hours. 
4) Following hydration the resident should be reminded to go to or be taken to the bathroom to promote urinary and bowel continence and reduce the risk of urinary tract infections.

5) Some time in both each morning and afternoon should include an activity that stimulates a mental and a physical activity.

6) Some period of the afternoon should contain a "quiet time". This does not necessarily mean a nap (although it could). This time would be when voices are at a minimum and calmness and relaxation abounds. For the Reflections group, this time is longer and includes a nap.

\section{Method}

\section{Implementation of MIR in Multiple Settings}

MIR, a fully comprehensive program, began as a psychosocial program for nursing homes developing dementia units. We considered the initial implementation of MIR and "Reflections" as a pilot or "demonstration of concept" project. A key issue at this point was whether an MIR program could be implemented in other residences not administered by its creators, and if so, what results might be obtained. Since its inception, the program has expanded to meet the needs of other skilled nursing residences as well as assisted living residences.

What followed was the installation of MIR with MBDP within 9 additional skilled nursing residences and 7 assisted living residences in Ohio by the MIR creation team. For each of the 9 skilled nursing and 7 assisted living residences, the MIR team provided customized programming based on the residence's individualized needs, size, population, geographic/cultural settings, challenges, staffing and goals. All residences had or added a separate area for a memory loss unit at the start of implementation of MIR.

\section{Results}

Table 1 and Table 2 show the results of data obtained from these dementia care residences over the course of a

Table 1. Percent (\%) reduction in use of medications wandering, agitation, and employee turnover before (1 year) and after (1 year) of MIR in memory units of Skilled Nursing Facilities (SNFs) and Assisted Living (AL) residences.

\begin{tabular}{|c|c|c|c|c|c|c|c|c|}
\hline \multirow{3}{*}{$\begin{array}{l}\text { Center } \\
\text { SNF1 }\end{array}$} & \multicolumn{3}{|c|}{ Medication Type } & \multirow[b]{2}{*}{ Hypnotics } & \multirow{3}{*}{$\begin{array}{c}\text { Wandering } \\
85\end{array}$} & \multirow{3}{*}{$\begin{array}{c}\text { Agitation } \\
85\end{array}$} & \multirow{2}{*}{\multicolumn{2}{|c|}{$\begin{array}{l}\text { Employee\#Residents } \\
\text { Turnover }\end{array}$}} \\
\hline & \multirow{2}{*}{$\begin{array}{c}\text { Anti-Psyc } \\
78\end{array}$} & \multirow{2}{*}{$\begin{array}{c}\text { Anti-Anx } \\
79\end{array}$} & \multirow{2}{*}{$\begin{array}{c}\text { Anti-Dep } \\
42\end{array}$} & & & & & \\
\hline & & & & 100 & & & 31 & 12 \\
\hline SNF2 & 75 & 76 & 40 & 100 & 85 & 85 & 31 & 14 \\
\hline SNF3 & 76 & 72 & 32 & 100 & 87 & 89 & 15 & 60 \\
\hline SNF4 & 78 & 73 & 41 & 100 & 79 & 86 & 10 & 12 \\
\hline SNF5 & 46 & 42 & 21 & 90 & 86 & 88 & 15 & 20 \\
\hline SNF6 & 56 & 51 & 32 & 89 & 75 & 76 & 15 & 12 \\
\hline SNF7 & 30 & 25 & 15 & 70 & 72 & 73 & 15 & 24 \\
\hline SNF8 & 50 & 48 & 35 & 95 & 88 & 90 & 20 & 10 \\
\hline SNF9 & 55 & 50 & 30 & 100 & 89 & 90 & 42 & 12 \\
\hline Average & 60 & 57 & 32 & 94 & 83 & 85 & 22 & \\
\hline AL1 & 60 & 55 & 40 & 100 & 75 & 80 & 15 & 12 \\
\hline AL2 & 58 & 55 & 35 & 100 & 95 & 95 & 12 & 10 \\
\hline AL3 & 20 & 20 & 15 & 90 & 85 & 85 & 12 & 8 \\
\hline AL4 & 60 & 58 & 41 & 100 & 87 & 90 & 15 & 30 \\
\hline AL5 & 60 & 59 & 45 & 100 & 90 & 90 & 15 & 15 \\
\hline AL6 & 60 & 60 & 46 & 100 & 91 & 92 & 15 & 15 \\
\hline AL7 & 42 & 65 & 60 & 100 & 87 & 90 & 15 & 15 \\
\hline Average & 53 & 53 & 40 & 98 & 87 & 89 & 14 & \\
\hline
\end{tabular}


Table 2. Percent (\%) increase of residents showing in weight gain, residents who began sleeping at night, and census; eating (\# of residents regaining ability to feed themselves), and ADLs (\# of residents who regained/improved one ADL), before (1 year) and after (1 year) of MIR in memory units of Skilled Nursing Facilities (SNFs) and Assisted Living (AL) residences.

\begin{tabular}{|c|c|c|c|c|c|c|}
\hline Center & Weight Gain & Sleeping & Census & Eating & $\mathrm{ADls}$ & \#Residents \\
\hline SNF1 & 100 & 90 & 20 & 2 & 12 & 12 \\
\hline SNF2 & 100 & 90 & 20 & 3 & 7 & 14 \\
\hline SNF3 & 99 & 97 & 26 & 8 & 36 & 60 \\
\hline SNF4 & 95 & 95 & 29 & 2 & 8 & 12 \\
\hline SNF5 & 96 & 89 & 15 & 4 & 10 & 20 \\
\hline SNF6 & 81 & 82 & 15 & 2 & 2 & 12 \\
\hline SNF7 & 80 & 80 & 15 & 6 & 12 & 24 \\
\hline SNF8 & 95 & 92 & 25 & 1 & 5 & 10 \\
\hline SNF9 & 85 & 90 & 14 & 2 & 8 & 12 \\
\hline Average & 92 & 89 & 20 & 3 & 11 & \\
\hline AL1 & 89 & 80 & 25 & 2 & 6 & 12 \\
\hline AL2 & 98 & 98 & 67 & 0 & 10 & 10 \\
\hline AL3 & 90 & 85 & 75 & 1 & 6 & 8 \\
\hline AL4 & 92 & 90 & 15 & 4 & 15 & 30 \\
\hline AL5 & 95 & 95 & 10 & 2 & 8 & 15 \\
\hline AL6 & 97 & 98 & 10 & 2 & 12 & 15 \\
\hline AL7 & 92 & 85 & 87 & 2 & 8 & 15 \\
\hline Average & 93 & 90 & 41 & 2 & 9 & \\
\hline
\end{tabular}

year after implementation of MIR in comparison with the same measures for the year previous to implementation of MIR. Much of the clinical data were collected from Skilled Nursing Facilities' (SNF) Directors of Nursing and Assisted Living (AL) Executive Directors. (Many of the ALs' Executive Directors were nurses.) Other data were collected by observation and staff interviews.

\subsection{Reductions of Negative Indices}

The results displayed in Table 1 demonstrate the capacity of MIR to reduce problematic outcomes related to both residents' quality of life and to factors important to dementia care residences' administrators. Medication reductions such as anti-psychotics, anti-anxiety, anti-depressants and hypnotics shown in Table 1 are based on an overall percentage reduction of each medication, based on monthly pharmacy tracking, before and after one year of programming. All showed reductions after implementation of MIR in all settings. This represents both a cost savings and a means of meeting demands from regulatory agencies for reducing medication usage.

Wandering and agitation reduction included tracking episodes per day by comparison before and after one year of programming. This was based on reporting from front-line staff. Finally, staff turnover was reduced in all facilities after implementation of MIR. Employee turnover was based on the percentage of turnover reduced before and after one year of programming. This makes sense from the point of view that reducing behaviors in residents such as agitation, wandering, and being awake at night would relate to reduced stress for staff members.

\subsection{Increases in Positive Indices}

Table 2 illustrates improvement in a number of important areas related to dementia care. Weight gain increases were based on percentage of residents who experienced appropriate weight gain. Staff also looked at intake records of residents, verifying consistency with the reported weight gain.

Increased night sleeping was reported by night shift staff giving results. This was based on percentage of residents who began sleeping at night, rather than being up at night and sleeping during the day. Third shift staff 
members reported in several centers that they could tell if the program had been appropriately implemented during the day by how well the residents slept that night or not.

Census improvement represents increase based on percentage before and after one year of programming. Again, census increased in all SNFs and ALs, a finding that further validates MIR both as a means of increasing quality of life for residents as well as a viable business model for administrators.

Eating data increases represent the actual number of residents who regained the ability to feed themselves. This was observed by staff and in many SNF's tracked in restorative nursing documentation. ADL data increases represent the actual number of residents who regained/improved at least one ADL, e.g., mobility, toileting, self-care and/or finger dexterity. This was observed by staff and in many SNF's tracked in restorative nursing documentation.

\subsection{Improvements in Indices Specific to SNFs}

Case Mix data were collected reflecting the actual increased RUGs number for the SNF centers, before and after one year of programming. All nine SNFs showed an increase in their RUGs number, ranging from 0.22 to 0.53 , with the average being 0.28 . This increase positively affects overall Medicaid rate in SNFs, resulting in increased income. Long term resident case mix scores can be positively increased with restorative nursing programming, which easily is incorporated into MIR, as we have noted previously.

Data were collected for Medicare Part B rehabilitation utilization for long-term SNF residents. This represents compensation given to SNFs by the Medicare program independent of income from short-term stay residents who are to receive rehabilitation for a brief period. In the nine SNFs implementing MIR, the increased percentage of long-term stay SNF residents receiving Medicare Part B services ranged from 27\% to 36\%, with an average across all SNFs of $32 \%$.

MIR implementation assists the staff in identifying the needs for therapy services. Within the MIR program, residents are able to be monitored consistently for their ADL abilities and changes, and observed changes can easily and appropriately become referral sources for therapy services. Related outcomes include increased ADL abilities and increased Part B revenue - a win-win for all, both management and the residents. As was the case with previous outcomes reported, improvements in RUGs case mix data and Medicare Part B utilization for long-term SNF residents represents changes from the previous year's outcomes compared to outcomes in the first year of MIR implementation.

\subsection{Aging in Place within ALs}

An important measure of the effect of implementing MIR for ALs is increasing "aging in place". This improvement number represents the average number of months per year that increased for residents to stay in the $\mathrm{AL}$ setting - thus "aging in place"-from before implementation of MIR to after implementation of MIR. Keeping residents within an AL enables more stability and less disruption in their lives for residents, as well as insuring a more constant source of income for ALs. Across the seven ALs, number of months of additional aging in place ranged from 2 months to 4 months, with an average of 3.2 months. This change also represents changes from the previous year's outcomes compared to outcomes in the first year of MIR implementation.

\section{Conclusions}

For overall ADL improvements, using a restorative nursing program within the daily MIR program had the best outcome. In many of the cases of improvement, the residents, particularly in the SNF's, were put on two restorative programs. Their programs were selected by the areas in which they needed the most improvement. Again, Montessori techniques proved to be an excellent non-pharmacological intervention in these restorative nursing programs particularly including self-care, eating and finger dexterity. When tracked as a restorative nursing program, the resident and facility both gain. The resident gains increased self-independence. SNF's have the ability, if tracked and documented appropriately; to increase their overall case mix score resulting in increased revenue.

$\mathrm{AL}$ residents benefit as well from MIR combined with MBDP. For example, the resident's "aging in place" time frame (being able to live in the same location without having to move) improves. The AL residence also gains in marketability when "aging in place" data improve, another systemic motivator for maintaining the program. 
MIR has been shown to have meaningful effects on measures relevant to administrators of long-term care residences and for residents as well. In so doing, MIR becomes a means of enhancing the importance of activities programming within these settings, while providing a method of enabling engaging activities to be delivered to residents throughout the day. Allowing residents to take part in a sustained program of engaging activities only will become a priority within long-term care when so doing aligns itself with systemic motivators, including income, effective marketing, and addressing stressors in the system such as responsive behaviors, especially those of importance to surveyors/inspectors (e.g. reduction of medication usage). Provision of MIR generates evidence of such an alignment, and as such may become an effective engine for culture change in long-term care environments.

"A social change of this type cannot come from the ideas or energies of individual reformers but from a slow and steady emergence of a new world in the midst of the old..." -Maria Montessori

\section{References}

[1] Camp, C.J. (2006) Montessori-Based Dementia Programming ${ }^{\mathrm{TM}}$ in Long-Term Care: A Case Study of Disseminating an Intervention for Persons with Dementia. In: Intrieri, R.C. and Hyer, L., Eds., Clinical Applied Gerontological Interventions in Long-Term Care, Springer, New York, 295-314.

[2] Roberts, G., Morley, C., Walters, W., Malta, S. and Doyle, C. (2015) Caring for People with Dementia in Residential Aged Care: Successes with a Composite Person-Centered Care Model Featuring Montessori-Based Activities. Geriatric Nursing, 36, 106-110. http://dx.doi.org/10.1016/j.gerinurse.2014.11.003

[3] Camp, C.J., Skrajner, M.J., Lee, M.M. and Judge, K.S. (2010) Cognitive Assessment in Late Stage Dementia. In: Lichtenberg, P.A., Ed., Handbook of Assessment in Clinical Gerontology, 2nd Edition, John Wiley \& Sons, New York, 523-547. http://dx.doi.org/10.1016/b978-0-12-374961-1.10020-x

[4] Cohen-Mansfield, J. (2015) Behavioral and Psychological Symptoms of Dementia. In: Lichtenberg, P.A., Mast, B.T., et al., Eds., APA Handbook of Clinical Geropsychology, Vol. 2: Assessment, Treatment, and Issues of Later Life, APA Handbooks in Psychology, American Psychological Association, Washington DC, 271-317. http://dx.doi.org/10.1037/14459-011

[5] Mast, B.T., Shouse, J. and Camp, C.J. (2015). Person-Centered Assessment and Intervention for People with Dementia. In: Lichtenberg, P.A., Mast, B.T., et al., Eds., APA Handbook of Clinical Geropsychology, Vol. 2: Assessment, Treatment, and Issues of Later Life, APA Handbooks in Psychology, American Psychological Association, Washington, DC, 319-339. http://dx.doi.org/10.1037/14459-012

[6] Zgola, J.M. (1987) Doing Things: A Guide to Programming Activities for Persons with Alzheimer's Disease and Related Disorders. Johns Hopkins University Press, Baltimore.

[7] Zgola J.M. (1999) Care That Works: A Relationships Approach to Persons with Dementia. Johns Hopkins University Press, Baltimore.

[8] Camp, C.J. (1999) Montessori-Based Activities for Persons with Dementia. Vol. 1, Menorah Park Center for Senior Living, Beachwood.

[9] Camp, C.J., Schneider, N., Orsulic-Jeras, S., Mattern, J., McGowan, A., Antenucci, V.M., Malone, M.L. and Gorzelle, G.J. (2006) Montessori-Based Activities for Persons with Dementia. Vol. 2, Menorah Park Center for Senior Living, Beachwood. 